\title{
The assessment of civil engineering heritage
}

\section{P. S. M. Cross-Rudkin, R. Cragg, P. Dunkerley, M. H. Gould and R. A. Otter}

\section{N. Gray, Department of Civil, Structural and Environmental Engineering, Trinity College, University of Dublin}

The importance of a formal assessment procedure for historical civil engineering works cannot be overstressed. With very little funding available for assessment, conservation and restoration, there is an urgent need for comparative ranking of structures so that limited funds can be prioritised. Rather than using a simple site-specific assessment, the use of a national or international system allows the relative importance of structures to be identified from the outset.

The UK is particularly well-served by county-based voluntary groups and organisations that can collect and record this type of data. In other European countries this is not the case, resulting in a need for a formalised response on a Europe-wide basis. In the first instance, funding should be made for assessment in order to produce national and international directories of historic structures. This will allow international, national, regional and local funding to be more appropriately allocated. Without a formal assessment procedure, assessment will be subjective, with local factors, both positive and negative being overemphasised.

Of the eight factors identified by the authors, condition is one of the most important in civil engineering terms, especially the safety of the structure, its state of dereliction and urgency for action. Aesthetics is a particularly broad term and difficult to quantify. However, it must take into consideration the importance of certain structures to landscape quality, such as mines, elevated sites or wide open spaces such as moorland.

The critical factors, namely safety and cost of restoration, often result in a conservative approach being taken that leads normally to demolition. First, the immediate problem of safety is the issue that frequently brings the structure to notice via complaints from the public. Second, the cost of surveying and pricing staged restoration (i.e. stabilisation of structure, prevention of further deterioration, and finally partial or full restoration) can be substantial. In practice, the simplest operation has been to demolish, unless the structure is of obvious national or local importance. So a formal national assessment procedure will identify and hopefully prevent the loss of important structures in the future.

Assessment is a huge undertaking as structures are so varied and widely distributed. Therefore, there is a need for unambiguous assessment procedures with appropriate training, handbooks, and harmonisation procedures for assessment and scoring. There are obvious difficulties in comparing the relative importance of different structures, especially if using different or modified assessment procedures as, according to the authors, the sub-panels are currently employing. Therefore unified assessment criteria, employing fully developed and annotated checklists, must be used. There is always a danger of setting an upper limit on the percentage of grade I or II structures permitted. This should be resisted as some categories of structures will be more at risk than others, requiring a greater proportion of the available funding. Assessments should be reviewed at least every five years, with more regular site visits to ensure that the structure has not significantly deteriorated. Where an immediate small investment may prevent serious and rapid deterioration of a structure, these cases should be examined and evaluated separately.

The four broad assessment categories (A, B, C and D) outlined by the authors may not be sufficiently discriminating. While reasons for not weighting factors were given, weighting allows a more balanced overall evaluation. The current system is still too subjective. There has to be cut-off points, and an impartial separation of two closely scored projects will only be possible if a numerical system is used. Also, certain assessment factors are clearly more important than others; so where a large number of factors are considered then weighting will provide more objectivity than the broad AAA, AAB scoring system proposed.

Transparency of assessment is vitally important, especially at a local level. Public consultation and open discussion of scores should be a vital part of the overall assessment procedure to ensure full data acquisition of sites and to gauge the local socio-economic importance of structures. Once assessment is complete, then it is important to separate directories into broad structure groups to ensure that some funding is allocated to all groups and not just one or two.

\section{Authors' reply}

The authors welcome the contribution from Professor Gray, which supports the general principles outlined in the paper. They would hope that their approach might be adopted in any more extended directories as envisaged by Professor Gray.

The unified system which he suggests was tried earlier (paragraph 8) and found to give unacceptable results. The 
authors have arrived at the present system after extended debate and experimentation, and believe that it is both transparent and capable of general use. They consider that to have more than four grades in factors of assessment or overall scoring would be unnecessarily complicated, and note that these four correspond with the grades used in listing in the United Kingdom. Opinions do vary about the relative importance of the eight factors. For this reason, and those given in the paper (paragraph 15), the authors adhere to their view that the present method of aggregation is to be preferred.

It might be noted that English Heritage is at present engaged in a Monuments Protection Programme which includes some of the features which Professor Gray mentions. 